\title{
A novel scavenger receptor 5-based antibiotic-independent selection method for generation of stable recombinant protein-producing mammalian cell lines especially suitable for proteins affecting cell adhesion
}

\author{
Juha Risto Matias Ojala, Timo Pikkarainen, Anna Domogatskaya, Karl Tryggvason, and Sergey Rodin \\ Divisions of Matrix Biology, Department of Medical Biochemistry and Biophysics, Karolinska Institute, \\ Stockholm, Sweden
}

BioTechniques 53:221-230 (October 2012) doi 10.2144/0000113936

Keywords: stable transfection; improvement of adhesion; recombinant proteins; mammalian cell lines

The establishment of stable recombinant protein-producing mammalian cell lines is an expensive, time-consuming, tedious procedure. In some cases, expressed recombinant proteins have adverse effects on host cell function, including cell adhesion. Based on the adhesive properties of SCARA5, a scavenger receptor (SR) of the class A SR family, we developed a method for selection of stable recombinant protein-producing cell clones that relies on an internal ribosome entry site (IRES) vector where the protein of interest is expressed in the same messenger RNA as SCARA5, resulting in improved adhesion and increased cell viability of recombinant protein-producing cells in serum-free media. This method does not depend on antibiotics, complicated selective cell culture media or equipment, and thus offers the advantages of being inexpensive, environmentally friendly, and simple.

Various approaches have been developed for large-scale recombinant protein production for protein crystallization and biotechnological purposes. Bacterial expression systems have proven to be reliable, fast, inexpensive, and easy to apply in high-throughput studies. In structural biology, approximately $90 \%$ of proteins have been produced using Escherichia coli $(1,2)$. Cell-free, yeast, baculoviral, and mammalian cell-based expression systems are also available and are often necessary for solving structures of mammalian proteins, since many have proven to be difficult to express in bacterial systems (1). In the biotechnology industry, more than $50 \%$ of approved therapeutic proteins are manufactured in mammalian expression systems $(3,4)$, the only choice when correct glycosylation of a recombinant protein is needed. Indeed, to ensure stability and function and to prevent immunological reactions, recombinant proteins used as biopharmaceuticals must closely resemble their native variants $(4,5)$.

For protein crystallization, most mammalian proteins are produced in transiently transfected human embryonic kidney (HEK) 293T, 293/EBNA, or 293S GnTI' cells $(6,7)$. Transient transfection systems are relatively inexpensive and diminish time-consuming selection steps for establishing stable clones $(1,8,9)$. An additional advantage of transient systems is that they do not require selection antibiotics, which are expensive and have to be destroyed after a production run. However, transient expression systems provide relatively low recombinant protein yields and require repeated transfections.

For protein crystallography and biotechnology, there is still a need for fast, highthroughput, low-cost selection methods for establishing stable, high-producing mammalian cell lines. In the biotechnology industry, clonality of protein-producing cells is often required. To date, many improvements have been made in protein yields by generating specific host cell lines (e.g., dihydrofolate reductase-deficient $\mathrm{CHO}$ cell line) and expression constructs, formulating various cell culture media, and improving bioprocess conditions $(2,3,10)$. However, current methods for establishing stable cell lines for large-scale protein production are still very time-consuming, laborious, relatively low-throughput, and dependent on specialized equipment or selection media with antibiotics. Costs and time needed for protein production runs may also increase, since there is often no guarantee that clones with the highest expression of a protein of interest are selected (11).

Scavenger receptors of the class A (SCARA) family are trimeric type II membrane proteins, which are able to bind diverse polyanionic ligands including modified low-density lipoprotein (LDL), polynucleotides, bacterial products, and modified extracellular matrix proteins (12-14). SCARA5 has a similar domain structure to the first identified member in the class, scavenger receptor A (SR-A, SCARA1), which has been shown to promote cell adhesion to serum-coated tissue culture dishes in a cation-independent manner $(15,16)$. In this article, we show how coexpression of SCARA5 can be advantageous for production of a recombinant protein with adverse effects on such an important host-cell function as its adhesive capacity. We also utilize the adhesion capacity of SCARA5 for developing a selection method for stable protein-producing cell clones. The selection method is simple, fast, and inexpensive, since there is no need for specialized equipment or expensive antibiotics. The method can 
A
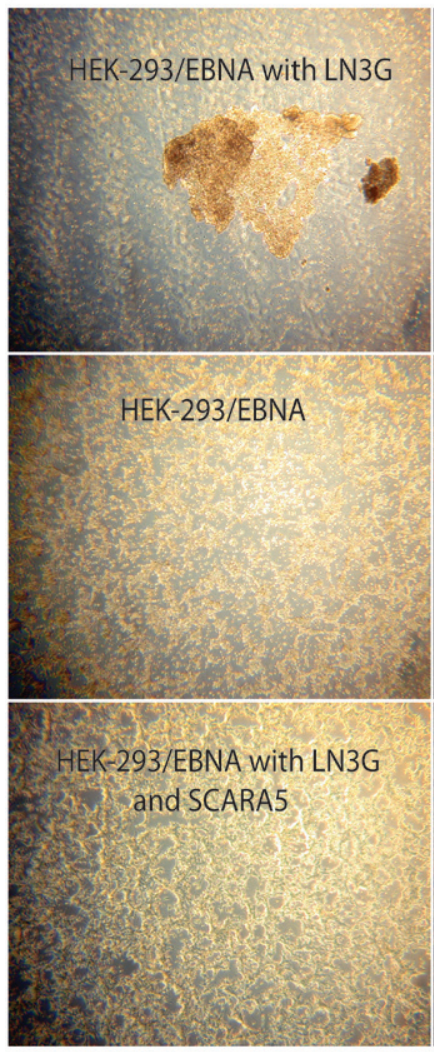
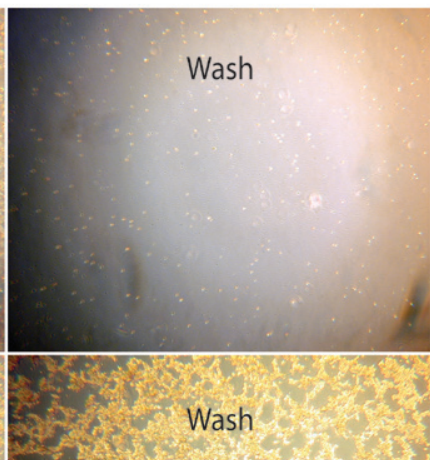

B

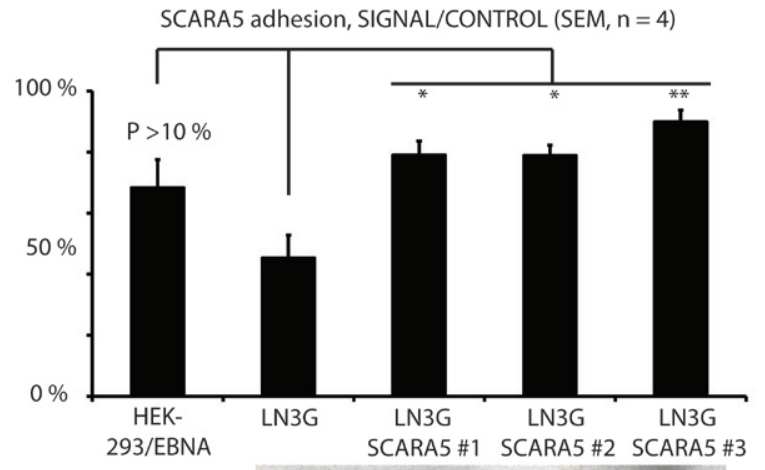

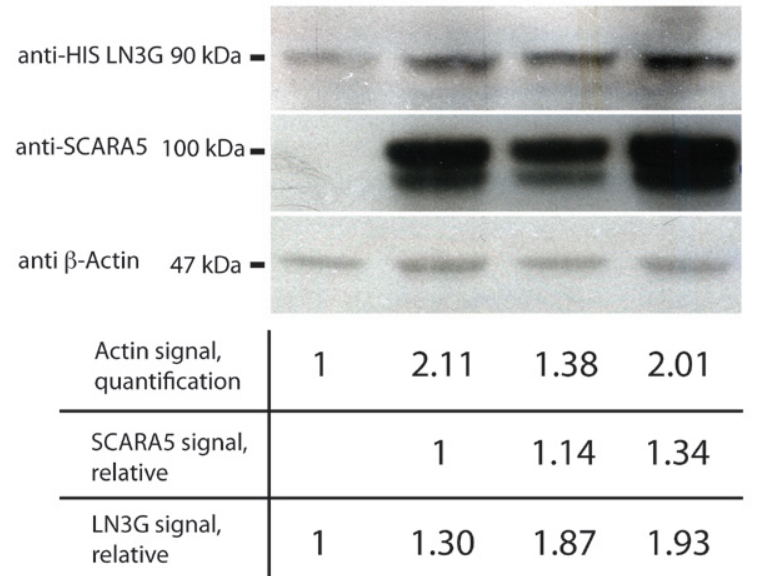

Figure 1. SCARA5 rescues weak adhesion of HEK-293/EBNA cells expressing LN3G. (A) Microphotographs of HEK-293/EBNA, a HEK-293/EBNA clone expressing LN3G, and a derivative of the latter cell type that also expresses SCARA5. Cells were cultured for 10 days in serum-free media, and the plates were photographed before and after PBS washes. The HEK-293/EBNA-LN3G cells of all 20 isolated clones easily detached from the plate during the washes. Parental HEK-293/EBNA cells and those expressing both LN3G and SCARA5 remained attached to the plates after the washes. (B) An adhesion assay for normal HEK-293/EBNA cells, an LN3G-expressing clone, and three LN3G/SCARA5-clones. The cell lines were also analyzed by Western blot analysis to quantify LN3G and SCARA5 expression. Double transfectants showed strongest adhesion. Western blot analyses of the cell lysates showed that the clone with highest SCARA5 expression exhibited the strongest adhesion to plastic. Interestingly, all the LN3G/SCARA5-clones expressed higher levels of LN3G than their parental LN3G-clones. SCARA5 and LN3G signals were quantified using $\beta$-actin signals for normalization of the sample input.

be applied to HEK-293 and CHO cells. The method also provides selection pressure for clones with high recombinant protein expression levels, which is highly desirable from both protein crystallographic and biotechnologic points of view.

\section{Materials and methods}

\section{LN3G-producing construct}

A 1.7-kb fragment encoding the $G$ subdomains $1-3$ (LN3G) of the mouse laminin $\alpha 3$ chain was PCR-amplified from a mouse small intestine cDNA library (BD Biosciences, Stockholm, Sweden) using the primers 5'-CGGCCTGCAGGGCCAGAGATGCTGCAAACAAGG-3' and 5'-GGGGTACCTTAATCTTGCAACAGCTGATTGACATTG-3' ${ }^{\prime}$ and cloned in frame after a cassette composed of the human TIMP-2 secretion signal sequence and a polyhistidine-tag in the pcDNA3.1/Zeo(-) vector (Invitrogen, Stockholm, Sweden). The plasmid was sequenced to confirm its identity.
Generation of LN3G-producing

HEK-293/EBNA cell clones

HEK-293/EBNA cells were transfected using the calcium-phosphate method with $40 \mu \mathrm{g}$ LN3G-expressing construct. Transfected cells were selected with $500 \mu \mathrm{g} / \mathrm{mL}$ Zeocin. Zeocin-resistant clones were tested for recombinant protein production using Western blot analysis with Penta-His antibodies (Qiagen, Sollentuna, Sweden).

Production of the LN3G protein

For the production of the $\mathrm{LN} 3 \mathrm{G}$ protein, $80 \%-90 \%$ confluent cultures of the selected cell clones grown in DMEM containing $10 \%$ fetal bovine serum (FBS) and $250 \mu \mathrm{g} / \mathrm{mL}$ Zeocin were washed twice with phosphate-buffered saline (PBS) to remove serum proteins and then fed with serum-free DMEM/F-12 (without phenol red) containing $250 \mu \mathrm{g} / \mathrm{mL}$ Zeocin. This washing step led to massive cell losses because all the selected clones exhibited low adhesion to cell culture plastic. Therefore, a new round of cell selection was performed after transfecting one of the clones with an expression construct encoding full-length SCARA5. After selection, clones were expanded and grown for 10 days in serumfree conditions for LN3G production. The medium was collected, centrifuged to remove cellular debris, and adjusted to 50 $\mathrm{mM}$ sodium phosphate, $\mathrm{pH} 7.5$, and 300 $\mathrm{mM} \mathrm{NaCl}$ (final concentrations) for recombinant protein purification. The protein was purified with Talon Metal Affinity Resins (BD Biosciences) according to the manufacturer's instructions.

\section{Adhesion assay}

Cells were plated at the density of 700 cells/ $\mathrm{mm}^{2}$ on two 96-well plates (four repeats per each clone) and were left to adhere for $1 \mathrm{~h}$ at $37^{\circ} \mathrm{C}$. Thereafter, cells on one of the plates were directly fixed for $20 \mathrm{~min}$ with $5 \%$ glutaraldehyde, washed, and stained with $0.1 \%$ crystal violet. These wells represented the initial number of cells. The other plate was washed several times with PBS, fixed, washed again, and stained as described above. After $1 \mathrm{~h}$ in crystal violet, excess dye was washed away, and the rest was extracted 
with $10 \%$ acetic acid and quantified by measuring optical density (OD) at $570 \mathrm{~nm}$. The results were expressed as a ratio of OD signal from the two plates. In some assays, cells were plated in the presence or absence of polyinosinic acid (poly I), a scavenger receptor ligand. After $1 \mathrm{~h}$ adhesion at $37^{\circ} \mathrm{C}$, cells were incubated with or without $5 \mathrm{mM}$ EDTA for $10 \mathrm{~min}$, followed by PBS washes, fixation, and crystal violet staining. The results were analyzed by Student's $t$-test. Values with $P<0.05$ were considered to be statistically significant.

\section{Construction of the IRES-SCARA5} selection vector

The AcGFP-coding sequence was removed from the pIRES2-AcGFP1 vector (Clontech, Stockholm, Sweden) and replaced by the SCARA5-encoding sequence amplified from a testis cDNA library (BD Biosciences) using the primers 5'-GCTCTAGTGTCGCGGCCG CACGCGTCAGGGGACAGTACA-3' and $5^{\prime}$-GATATCCACAACCATGGACAACAAAGCC-3'. This treatment left the multiple cloning sites between the cytomegalovirus (CMV) promoter and the internal ribosome entry site (IRES) sequence available for cloning of any protein of interest. In this study, we inserted enhanced green fluorescent protein (EGFP) into this site (GFP-IRES-SCARA5 plasmid). In this construct, the NheI, XhoI, SacI, and PstI sites within the multiple cloning site were no longer available for cDNA insertion in the parental IRES-SCARA5 vector, since the SCARA5 cDNA contains recognition sites for these enzymes.

Selection of stable protein-producing cells with the use of SCARA5

HEK-293/EBNA cells were transfected with the GFP-IRES-SCARA5 vector, and propagated for 10 days in order to get rid of transient expression of GFP and SCARA5. Thereafter, the cells were trypsinized, trypsin was inactivated with FBS, and cells were washed several times with PBS to remove serum proteins. They were then resuspended in DMEM with $0.2 \%$ heat-inactivated albumin (plating medium), and plated at a density of 700 cells $/ \mathrm{mm}^{2}$ on gelatin-coated glasses $(30$ $\mathrm{mm}$ in diameter; VWR International $\mathrm{AB}$, Stockholm, Sweden) in 6-well plates. For coating, glasses were incubated overnight with $0.01 \%$ gelatin in PBS. Cells were allowed to adhere for $45 \mathrm{~min}$ at $37^{\circ} \mathrm{C}$, after which nonadherent cells were washed away by dipping the glasses several times in PBS. Attached cells were trypsinized and analyzed by flow cytometry for GFP expression.
A
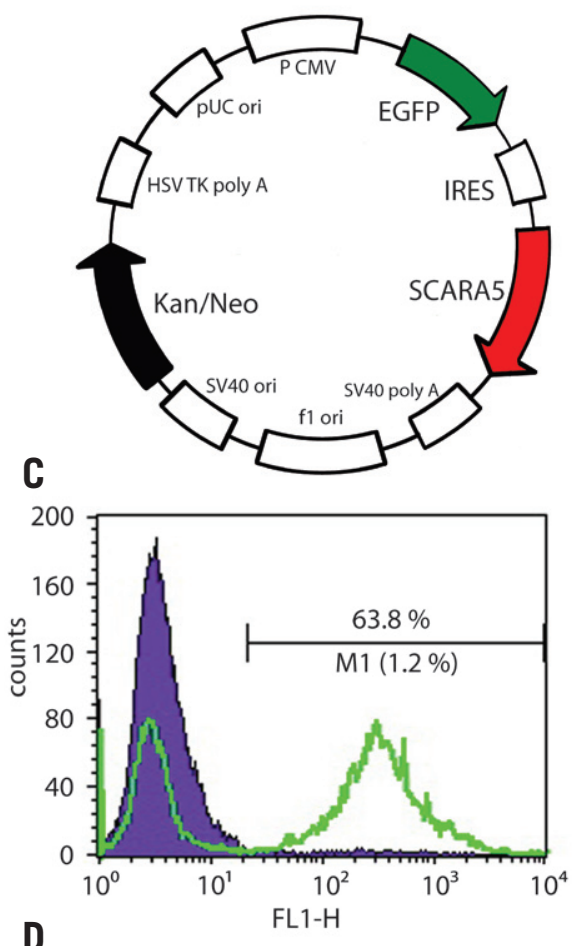

B

1. $80 \%$ of the transfected cells express SCARA5 and the protein of interest, EGFP

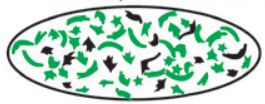

2. After 10 days in culture trancient expression is lost, $\sim 1.0 \%$ positive cells

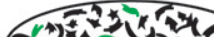

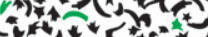
N. $=4+\infty+\infty$

3. Trypsinize and wash at least 3 times to remove serum 4. Plate the cells on gelatin coated glasses on a 6 well plate

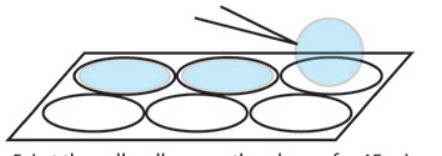

5. Let the cells adhere on the glasses for $45 \mathrm{~min}$

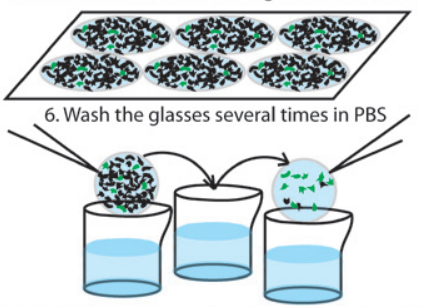

7. Trypsinize and plate for subcloning or use as a pool ( $60 \%$ positive cells)

8. Repeat the selection if desired

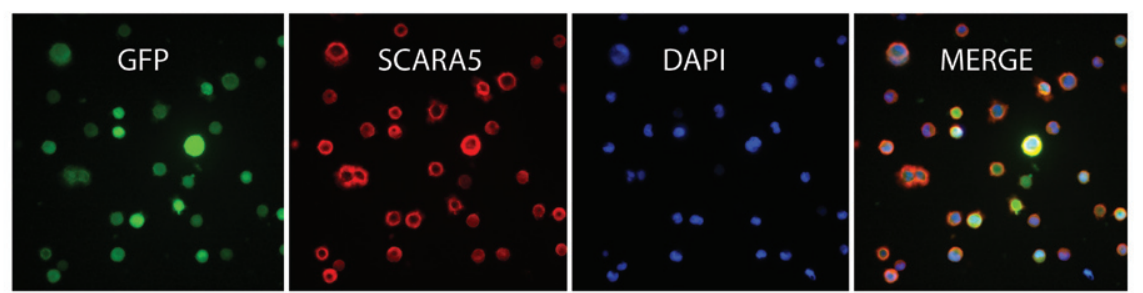

Figure 2. Selection of stable cell lines through the adhesive capacity of SCARA5. (A) Schematic of the IRES-SCARA5 selection vector. To generate the vector, the AcGFP1-encoding sequence downstream of the IRES sequence in the pIRES2-AcGFP1 vector was first replaced by the SCARA5-encoding sequence. Then EGFP was inserted into the multiple cloning site downstream of the CMV promoter. (B) The selection protocol. Transfected cells were grown for at least 10 days to get rid of transient expression. Cells were detached by trypsin treatment, washed, and plated onto gelatin-coated glass for 45 min at $37^{\circ} \mathrm{C}$. After several washes in PBS, adherent cells were plated sparsely for the isolation of individual clones or directly used as a pool for protein production. (C) FACS analysis shows that selection increased the number of GFP-positive cells from 1.2\% (purple) to 63.8\% (green). (D) Immunofluorescence staining of the selected cells. GFP- and SCARA5-positive cells are shown in green and red, respectively. Cell nuclei are visualized by DAPI staining (blue). A majority of cells is positive for both SCARA5 and GFP.

Western blot and densitometry analysis To confirm the expression of both LN3G and SCARA5, confluent monolayers of cells were washed twice with ice-cold PBS and lysed on ice in SDS loading buffer (Life Technologies, Carlsbad, CA, USA). The lysates were boiled and pushed through a $27 \mathrm{G}$ needle to shear the DNA. Polypeptides were separated on NuPAGE $4 \%-12 \%$ Bis-Tris gels (Life Technologies) under denaturing conditions and transferred to PVDF membranes. Thereafter, the membranes were incubated with mouse anti-penta-HIS antibodies (2 $\mu \mathrm{g} / \mathrm{ml}$; Qiagen) for detection of LNG3, or rabbit antibodies against SCARA5 $(2 \mu \mathrm{g} / \mathrm{ml})$ or b-actin (0.1ug/ml; ABcam, Cambridge, UK). Chemiluminescent horseradish perox- idase (HRP) substrate was used for visualization after incubation of the membranes with HRP-conjugated secondary antibodies. Films were scanned and analyzed using ImageJ (U.S. National Institutes of Health, Bethesda, MD, USA; http://imagej.nih. gov/ij/). Between staining procedures, the membranes were stripped by incubation in $100 \mathrm{mM} 2$-mercaptoethanol, 2\% SDS, $62.5 \mathrm{mM}$ Tris-HCl, $\mathrm{pH}$ 6.7, for $30 \mathrm{~min}$ at $50^{\circ} \mathrm{C}$.

Immunofluorescent staining

In some cases, we also used immunofluorescence to examine SCARA5 expression in the remaining cells attached to the glass after washing. Cells were fixed with 
4\% paraformaldehyde in PBS for 10 min, blocked for $30 \mathrm{~min}$ at room temperature with 2\% BSA in PBS solution, and stained for $1 \mathrm{~h}$ at room temperature with polyclonal rabbit antobodies $(2 \mu \mathrm{g} / \mathrm{ml})$ against SCARA5. After PBS washes, the cells were incubated for $15 \mathrm{~min}$ with Alexa Fluor 568-conjugated secondary antibodies (Life Technologies) and nuclear marker 4',6-diamidino-2phenylindole (DAPI). After the final washes, the glasses were mounted and examined under a fluorescence microscope.

Flow cytometry

GFP-IRES-SCARA5-transfected HEK-293/EBNA cells were analyzed by flow cytometry before and after the selection step. Cells were detached from plates or glasses by a trypsin/EDTA treatment, dissociated into a single-cell suspension by pipeting, resuspended in ice-cold fluorescence-activated cell sorting (FACS) buffer ( $2 \%$ FBS, $0.1 \%$ sodium azide in Hank's buffer), and analyzed using FACSCalibur Flow Cytometer (BD Biosciences). Data were analyzed with CellQuest software (BD Biosciences).

\section{Results and discussion}

Using the calcium-phosphate transfection method with a subsequent antibioticsbased selection step, we generated stable HEK-293/EBNA cell lines producing a recombinant laminin $\alpha 3$ chain fragment encompassing three $\mathrm{C}$-terminal $\mathrm{G}$ domains (LN3G) representing the main adhesive site of laminin-332 (17). However, cells of all 20 isolated clones selected for production of this $70-\mathrm{kD}$ a protein showed significantly reduced adhesion to cell culture plastic; cells easily detached and died, especially under serum-free conditions. This made it impossible to wash the cells to remove serum proteins for refeeding with serumfree media, which seriously complicated purification of the protein. In an attempt to circumvent this problem, we transfected an LN3G-expressing cell line with a plasmid encoding full-length SCARA5, a class A SR, that we previously found to promote cell adhesion to cell culture plastic (unpublished data). We established several stable double-transfected cell lines and cultured them for 10 days in serum-free conditions. Indeed, the double transfectants showed higher adhesion and viability than cells expressing LN3G alone (Figure 1A). Similar findings were obtained when comparing LN3G-expressing clones to clones from simultaneous transfection of LN3G- and SCARA5-encoding plasmids (data not shown). In order to quantify cell adhesion, we performed an adhesion assay
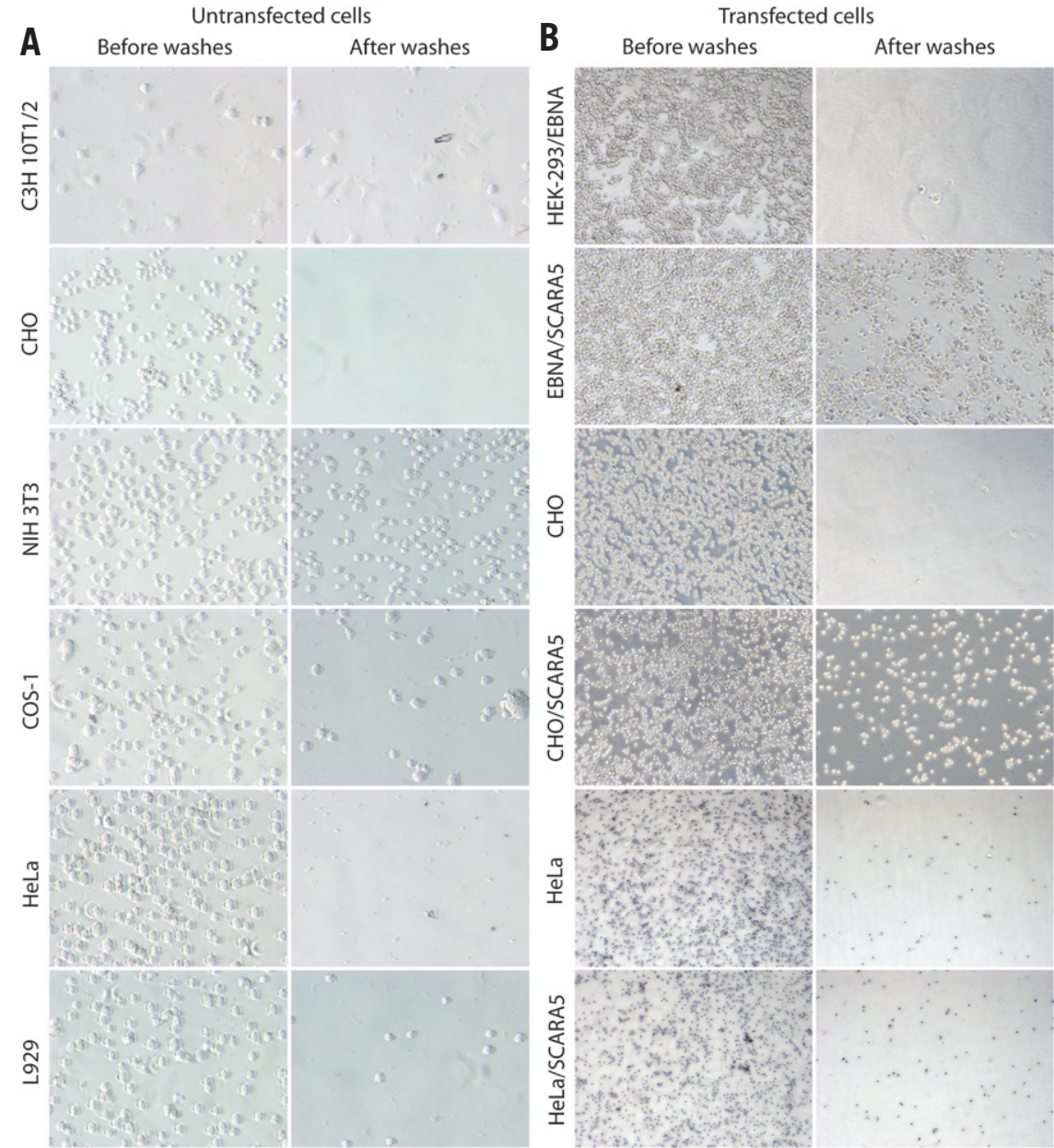

Figure 3. Screening of common cell lines for compatibility with our selection assay. (A) Microphotographs of untransfected C3H 10T1/2, CHO K1, NIH 3T3, COS-1, HeLa, and L929 cells before and after washing with PBS. Cells were plated onto gelatin-coated glass for $45 \mathrm{~min}$, after which nonadherent cells were removed by washing several times with PBS. Photographs were taken before and after the washes. (B) Microphotographs of HEK-293/EBNA, CHO, and HeLa cells transfected either with an empty vector control or the IRES-SCARA5 selection vector (depicted as EBNA/SCARA5, CHO/SCARA5, and HeLa/ SCARA5, respectively). The cells were plated and treated as described in panel A. Microphotographs were taken before and after washing with PBS.

on cell culture plastic with double and single transfectants, along with the parental HEK-293/EBNA cells. While adhesion of LN3G-expressing cells to plastic was significantly reduced when compared with untransfected cells, clones coexpressing LN3G and SCARA5 exhibited equal or increased adhesion compared with the single trasfectants (Figure 1B). Western blot analysis showed that double transfectants expressed equal or even higher levels of LN3G than LN3G-single transfectants. Furthermore, there seemed to be a correlation between the level of SCARA5 expression and the adhesive capacity, which, in turn, correlated with LN3G expression level (Figure 1B). Improved adhesion allowed us to easily wash the cells with PBS before changing the media to serum-free DMEM/F-12, as well as helped keep the cells viable for several days, leading to good yields of the $\mathrm{LN} 3 \mathrm{G}$ protein.
Motivated by these findings and our unpublished data that SCARA5 promoted cell adhesion not only to cell culture plastic but also to surfaces coated with gelatin, we developed a new antibiotic-free selection method for establishing stable cell clones based on the adhesive properties ofSCARA5. Using GFP as a test protein, we constructed a vector where GFP and SCARA5 were expressed in the same messenger RNA with the help of an IRES sequence (Figure 2A). HEK-293 cells were transfected with the vector and were first grown for 10 days in the absence of selective antibiotics to remove transient expression of SCARA5 and GFP. Thereafter, the cells were detached using a trypsin-EDTA solution, washed to remove serum proteins, plated on gelatincoated glass for $45 \mathrm{~min}$ to allow SCARA5mediated adhesion, and washed with PBS to remove non-adherent untransfected cells. The scheme of the selection protocol 


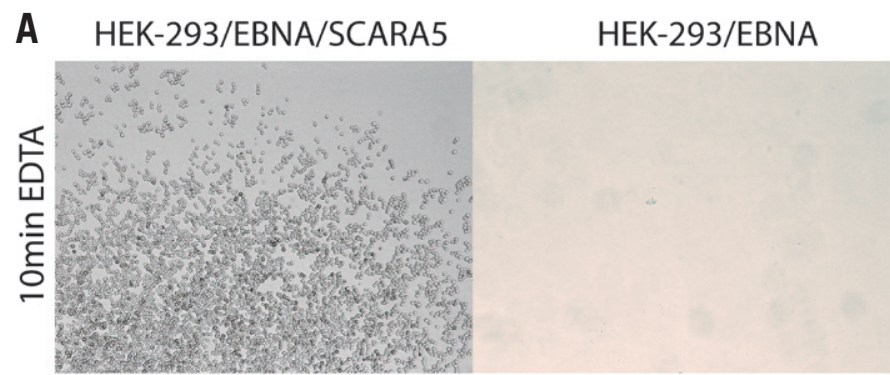

B

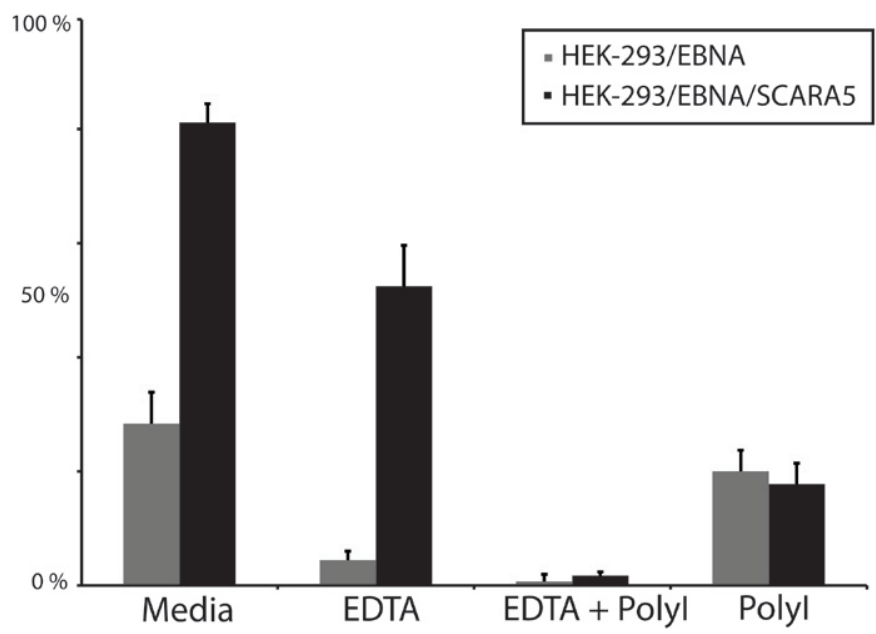

Figure 4. The effect of poly I and EDTA on SCARA5-mediated adhesion. (A) Cell culture dishes of adherent HEK-293/EBNA cells and their SCARA5-transfected derivatives were photographed after a 10-min EDTA treatment and PBS washes, which completely removed the parental cells. (B) An adhesion assay in which the cells were plated for $1 \mathrm{~h}$ in the presence or absence of poly I, a scavenger receptor ligand, followed by PBS washes, and a brief incubation with or without EDTA. The results are expressed as a percentage of a signal obtained from a control plate with no treatments and washes. SCARA5-positive cells showed avid adhesion even after the treatment with EDTA. However, the presence of poly I during the 1-h adhesion period blocked SCARA5-mediated adhesion.

is shown in Figure 2B. Flow cytometric analysis was used to quantify the proportion of GFP-positive cells prior to and after the selection. Forty to 60-fold increase in the number of GFP-positive cells was achieved by the method, and up to $65 \%$ of the cells were positive for this test protein, making subsequent generation and selection of highexpressing stable clones easy (Figure 2C). We also stained some glasses by immunofluorescence for SCARA5 after the selection procedure. Consistent with the flow cytometry results, a large majority of the cells still attached to the glass were positive for both GFP and SCARA5 (Figure 2D).

To determine if the method can be used with cell lines other than HEK-293 cells, we selected a panel of commonly used cell lines and studied their adhesion to gelatincoated glass. Fibroblast-like cell lines $\mathrm{C} 3 \mathrm{H}$ 10T1/2, L929, and NIH 3T3 exhibited binding to this surface and were therefore found unsuitable for this selection method (Figure 3A). Indeed, we have previously shown that cells of these lines express endogenous SCARA5 (unpublished data). Even without endogenous SCARA5 expression (not shown), COS-1 cells were able to bind to the gelatin-coated surface. However, $\mathrm{CHO}$ and HeLa cells exhibited no binding (Figure 3A). Similar to HEK-293/EBNA cells, transfection with SCARA5 significantly increased adhesion of $\mathrm{CHO}$ cells to the gelatin-coated surface (Figure 3B). This is a relevant finding, since $\mathrm{CHO}$ and HEK-293 cells are the two most common mammalian cell lines used for production of recombinant proteins $(3,10)$. On the other hand, transfection of HeLa cells with the IRES-SCARA5 vector did not significantly increase adhesion compared with cells transfected with an empty control plasmid, indicating that the new selection method is not suitable for this cell line.

The structurally similar scavenger receptor SR-A has been shown to mediate adhesion through a cation-independent mechanism (15), so we tested if SCARA5 had a similar property. Indeed, we found that adherent SCARA5-expressing HEK-293/EBNA cells, but not the parental cells, remained attached to cell culture plastic after EDTA treatment (Figure 4A). Pretreatment of the SCARA5-expressing
HEK-293/EBNA cells with a putative scavenger receptor ligand poly I (Figure 4B) blocked adhesion, proving that it was indeed mediated by SCARA5. This might be useful for microcarrier cultures developed for anchorage-dependent cells. For instance, coating of the microcarriers with this polyanionic molecule might decrease shear stress-induced cell loss. Poly I could be used as a substratum as an alternative to gelatin, and EDTA treatment to release untransfected cells.

In conclusion, this study shows that ectopic expression of SCARA5 in mammalian cell lines commonly used for large-scale protein production can improve the adhesive properties and viability of cells in serum-free culture conditions, thereby making it possible to produce proteins with adverse effects on host cells. Furthermore, our results indicate that SCARA5 can be used as a novel selection molecule for the cloning of stable recombinant protein-producing cell lines. The selection process has proven to be fast and inexpensive, does not require specialized equipment or expensive antibiotics, and provides selection pressure toward highexpressing clones. This method also allows researchers to minimize possible environmental pollution with antibiotics that contribute to antibiotic resistance of bacteria, which is a growing public health concern (18).

Similar to Sleiman (19) and DeMaria et al. (20), we utilized an IRES site to express a protein of interest on the same mRNA as the molecule used for selection. In our method, there is no need to stain the cells with antibodies or use FACS for sorting and selecting high-producing clones $(19,20)$, but these steps can be applied if desired.

Although we used the adhesive capacity of SCARA5 for cell selection, we believe that other adhesion molecules, such as other scavenger receptors and lectins, may also be used as selection tools for generating stable protein-producing cell lines. Since different sugars serve as ligands to lectins (21), a potentially large number of lectin/scavenger receptor combinations could be used for selection when many proteins or proteins with multiple subunits are expressed in the same cell (17).

\section{Acknowledgments}

This work was supported by grants to K.T. from the Knut and Alice Wallenberg Foundation and The Swedish Research Council.

\section{Competing interests}

The authors declare no competing interests. 


\section{References}

1. Aricescu, A.R., W. Lu, and E.Y. Jones. 2006. A time- and cost-efficient system for highlevel protein production in mammalian cells. Acta Crystallogr. D Biol. Crystallogr. 62:12431250 .

2. Nettleship, J.E., R. Assenberg, J.M. Diprose, N. Rahman-Huq, and R.J. Owens. 2010. Recent advances in the production of proteins in insect and mammalian cells for structural biology. J. Struct. Biol. 172:55-65.

3. Zhu, J. 2012. Mammalian cell protein expression for biopharmaceutical production. Biotechnol Adv. 30:1158-1170.

4. Andersen, D.C. and L. Krummen. 2002. Recombinant protein expression for therapeutic applications. Curr. Opin. Biotechnol. 13:117-123.

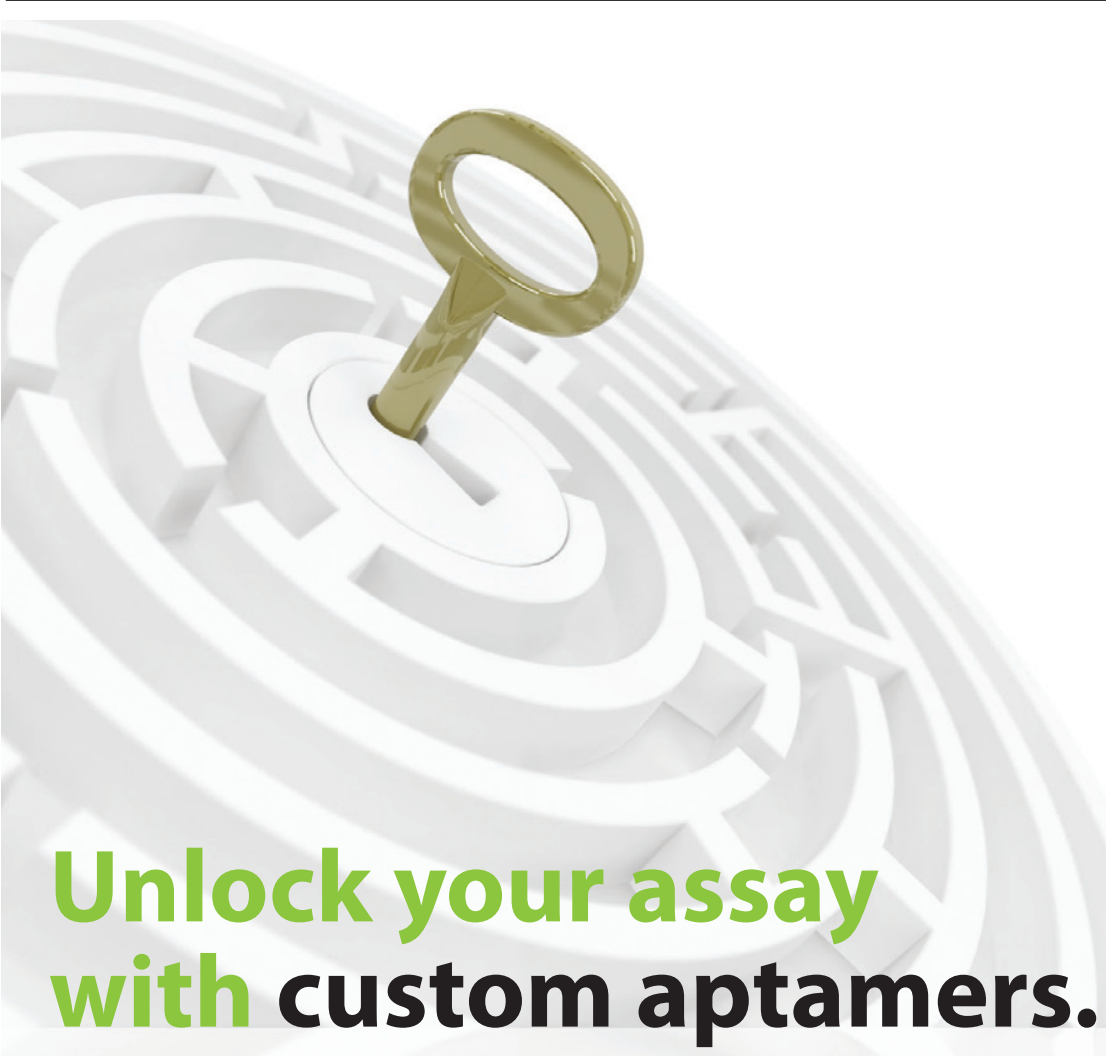

For more information on aptamers and their

applications visit

The Aptamer Lounge www.aptamers.com

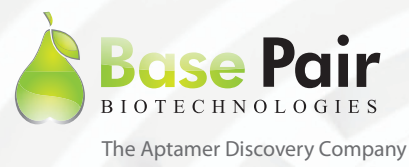

Base Pair Biotechnologies, Inc. 8058 El Rio St. • Houston, TX 77054 (978) 609-4199 • info@basepairbio.com
5. Sanchez, S. and A. Demain. 2012. Special issue on the production of recombinant proteins. Biotechnol Adv. 30:1100-1101.

6. Fliedl, L. and C. Kaisermayer. 2011. Transient gene expression in HEK293 and vero cells immobilised on microcarriers. J. Biotechnol. 153:15-21.

7. Zhao, Y., B. Bishop, J.E. Clay, W. Lu, M. Jones, S. Daenke, C. Siebold, D.I. Stuart, et al. 2011 Automation of large scale transient protein expression in mammalian cells. J. Struct. Biol. 175:209-215.

8. Geisse, S. and M. Henke. 2005. Large-scale transient transfection of mammalian cells: a newly emerging attractive option for recombinant protein production. J. Struct. Funct. Genomics 6:165-170.
9. Raymond, C., R. Tom, S. Perret, P. Moussouami, D. L'Abbe, G. St-Laurent, and Y. Durocher. 2011. A simplified polyethylenimine-mediated transfection process for large-scale and highthroughput applications. Methods 55:44-51.

10. Kim, J.Y., Y.G. Kim, and G.M. Lee. 2012. $\mathrm{CHO}$ cells in biotechnology for production of recombinant proteins: current state and further potential. Appl Microbiol Biotechnol. 93:917930

11. Browne, S.M. and M.Al-Rubeai. 2007. Selection methods for high-producing mammalian cell lines. Trends Biotechnol. 25:425-432.

12. Arredouani, M., Z. Yang, Y. Ning, G. Qin, R. Soininen, K. Tryggvason, and L. Kobzik. 2004. The scavenger receptor MARCO is required for lung defense against pneumococcal pneumonia and inhaled particles. J. Exp. Med. 200:267272.

13. Elomaa, O., M. Kangas, C. Sahlberg, J. Tuukkanen, R. Sormunen, A. Liakka, I. Thesleff, G. Kraal, and K. Tryggvason. 1995. Cloning of a novel bacteria-binding receptor structurally related to scavenger receptors and expressed in a subset of macrophages. Cell 80:603-609.

14. Gordon, S. 2002. Pattern recognition receptors: doubling up for the innate immune response. Cell 111:927-930.

15. Fraser, I., D. Hughes, and S. Gordon. 1993. Divalent cation-independent macrophage adhesion inhibited by monoclonal antibody to murine scavenger receptor. Nature 364:343346.

16. Jiang, Y., P. Oliver, K.E. Davies, and N. Platt. 2006. Identification and characterization of murine SCARA5, a novel class A scavenger receptor that is expressed by populations of epithelial cells. J. Biol. Chem. 281:1183411845.

17. Miner, J.H. and P.D. Yurchenco. 2004. Laminin functions in tissue morphogenesis. Annu. Rev. Cell Dev. Biol. 20:255-284.

18. Levy, S.B. and B. Marshall. 2004. Antibacterial resistance worldwide: causes, challenges and responses. Nat. Med. 10:S122-S129.

19. Sleiman, R.J., P.P. Gray, M.N. McCall, J. Codamo, and N.A. Sunstrom. 2008. Accelerated cell line development using two-color fluorescence activated cell sorting to select highly expressing antibody-producing clones. Biotechnol. Bioeng. 99:578-587.

20. DeMaria, C.T., V. Cairns, C. Schwarz, J. Zhang, M. Guerin, E. Zuena, S. Estes, and K.P. Karey. 2007. Accelerated clone selection for recombinant CHO CELLS using a FACSbased high-throughput screen. Biotechnol. Prog. 23:465-472.

21. Sharon, N. 2007. Lectins: carbohydrate-specific reagents and biological recognition molecules. J. Biol. Chem. 282:2753-2764.

Received 20 June 2012; accepted 24 August 2012

Comparable affinity and specificity

- We are not limited by non toxic or non immunogenic targets

You can have complete intellectual property to your aptamer if desired

Address correspondence to Karl Tryggvason, Divisions of Matrix Biology, Department of Medical Biochemistry and Biophysics, Karolinska Institute, SE-171 77 Stockholm. Email: karl.tryggvason@ki.se

To purchase reprints of this article, contact:

biotechniques@fosterprinting.com 